Marquette University

e-Publications@Marquette

Speech Pathology and Audiology Faculty Research

and Publications

Speech Pathology and Audiology, Department of

$1-1-2008$

A Comparison of Oral Motor and Production Training for Children with Speech Sound Disorders

Karen Forrest

Indiana University - Bloomington

Jenya Iuzzini-Seigel

Marquette University, jenya.iuzzini-seigel@marquette.edu

Published version. Seminars in Speech and Language, Vol. 29, No. 4 (2008): 304-311. DOI. (C) 2008

Thieme Medical Publishers Inc. Used with permission.

Jenya Iuzzini was affiliated with Indiana University at the time of publication. 


\title{
A Comparison of Oral Motor and Production Training for Children with Speech Sound Disorders
}

\author{
Karen Forrest, Ph.D., ${ }^{1}$ and Jenya luzzini, M.A. ${ }^{1}$
}

\section{ABSTRACT}

Despite the many debates about the usefulness of nonspeech oral motor exercises (NSOMEs) in the treatment of speech disorders, few controlled experiments have evaluated their efficacy in the remediation of phonological/articulatory disorders (PADs). More importantly, the relative effect of NSOMEs compared with traditional production treatment (PT) has not been established. The current study employed an alternating treatment design to evaluate changes in production of sounds targeted by NSOMEs and PT in nine children with PAD. Each subject received treatment on two linguistically distinct sounds in which one sound was treated with NSOMEs and the second sound was targeted with PT. The difference in treatment efficacy, measured as the percentage change in target production for NSOMEs versus PT, was compared using a paired $t$ test. Because NSOMEs typically are used to ready a child for subsequent PT, comparison of PT treatment accuracy was made between NSOME-first and PT-first sessions. Results demonstrated a statistically significant effect of treatment type with greater production gains with PT compared with NSOMEs. Further, no facilitative effect of NSOMEs on PT was noted; however, the choice of distinct treatment targets may have contributed to this null effect. Although additional investigation is warranted, the current investigation does not support the efficacy of NSOMEs in the treatment of PAD.

KEYWORDS: Phonological/articulatory disorders, nonspeech oral motor exercises, production treatment

${ }^{1}$ Department of Speech and Hearing Sciences, Indiana University, Bloomington, Indiana.

Address for correspondence and reprint requests: Karen Forrest, Ph.D., Department of Speech and Hearing Sciences, Indiana University, Bloomington, IN 47405 (e-mail: kforrest@indiana.edu).

Controversies Surrounding Nonspeech Oral Motor
Exercises for Childhood Speech Disorders; Guest Editor, Gregory L. Lof, Ph.D.

Semin Speech Lang 2008;29:304-311. Copyright (C) 2008 by Thieme Medical Publishers, Inc., 333 Seventh Avenue, New York, NY 10001, USA. Tel: +1(212) 5844662.

DOI 10.1055/s-0028-1103394. ISSN 0734-0478. 
Learning Outcomes: As a result of this activity, the reader will be able to (1) explain the rationale for the current study comparing the relative efficacy of NSOMEs and traditional production therapy (PT) on speech sound learning in children; (2) summarize the methods and results of the study; and (3) evaluate the implications of the study, and its potential weaknesses, for the use of NSOMEs in the remediation of speech sound disorders (SSDs) in children.

The use of nonspeech oral motor exercises (NSOMEs) to treat children's speech disorders has received extensive scrutiny within the past decade. ${ }^{1-3}$ Investigations of the usefulness of NSOMEs have addressed numerous issues, including the definition ${ }^{3}$ and delineation of NSOME procedures, ${ }^{2,4,5}$ the theoretical rationale for applying these techniques to speech remediation, ${ }^{3}$ the populations for whom NSOMEs are appropriate, ${ }^{3-7}$ and, to a lesser extent, the efficacy of the procedures in effecting improvement of impaired speech. Each of these areas helps clarify how and whether NSOMEs are useful adjuncts to therapy for children with speech disorders.

By definition, speech is a motor behavior that engages multiple levels of movement control. As such, the case can be made that all speech treatment incorporates oral motor exercise; ${ }^{4,8}$ however, such a perspective is beyond the realm of experimental investigation of treatment efficacy because the heterogeneity introduced by the multitude of independent variables (i.e., all treatments for all types of speech disorders) does not allow for a reliable index of their impact on a single dependent measure (i.e., accuracy of speech produced). For that reason, nonspeech and speech movement protocols must be dissociated to determine which procedures are most efficacious in changing disordered speech production. Therefore, the current investigation assumes Weismer's ${ }^{3}$ definition of NSOMEs as "any performance task, absent phonetic goals, in which structures of the speech mechanismespecially those of the upper airway" (p. 319) are used.

The rationale for using NSOMEs to improve speech production, especially in children with motor disorders, is quite appealing. For example, children with motor disorders may experience weakness or tone abnormalities that some clinicians believe need to be addressed before coordinated movements for speech can be expected. Because NSOMEs target these motor parameters, improved speech might be predicted from increased strength or tonal normalization. Further, some clinicians believe that the complex, rapid movements that characterize speech production may be learned more easily if they are isolated or simplified, and, once these patterns are mastered, the speech gestures can be reconstructed from their component parts. In addition, some believe that NSOMEs may represent earlier occurring movement patterns that naturally serve as a foundation for speech production. A final proposal by some clinicians for using NSOMEs is that they can orient a child to the mouth as a prelude to working on phoneme production. ${ }^{7}$ Populations who are thought to benefit from these activities include those with articulation disorders, ${ }^{4,9}$ childhood apraxia of speech, ${ }^{5}$ and various forms of dysarthria. ${ }^{4-6,9}$

Information presented by other researchers elsewhere in this journal ${ }^{10,11}$ contradict these rationales for the use of NSOMEs as a means of improving speech motor control (see also Forrest $^{6}$ ) both in neurologically healthy individuals and those with motor disorders. As will be presented, anatomical and physiological evidence, combined with previous experimental data from other disciplines, explain why and how NSOMEs will not change speech sound productions.

Although general principles of motor control $^{12-14}$ as well as physiological constraints of the oral motor system ${ }^{8,9}$ do not support the transfer of NSOME skills to speech, there are very few empirical evaluations of the impact of these procedures on speech learning (for reviews of these studies, see Ruscello ${ }^{15}$ ). Extant studies in peer-reviewed journals are equivocal in their endorsement of NSOMEs in the treatment of speech disorders. For example, Overstake ${ }^{16}$ monitored changes in /s/ production in two groups of children with tongue thrust who were treated with an unspecified 
swallowing therapy or a combination of swallowing treatment and production training $(\mathrm{PT})$ of $/ \mathrm{s} /$. A large majority of children who received only swallowing treatment improved their production of /s/ in conversational speech. Surprisingly, a smaller percentage of children who received both swallowing and speech therapy demonstrated improved articulation.

Christensen and Hanson ${ }^{17}$ used a controlled experimental design to investigate whether oral myofunctional therapy has a facilitative effect on subsequent articulation therapy for $/ \mathrm{s} /$ and $/ \mathrm{z} /$. Ten children between the ages of 5;8 and 6;9 (years; months), all with anterior tongue thrust and severe frontal lisps, were included in the study. One group of children received only articulation therapy for 14 weeks, and the second group of children received 6 weeks of myofunctional therapy for tongue thrust followed by 8 weeks of alternating myofunctional and articulation therapy. Comparison of pre- and post-treatment production of sibilants and performance on an articulation test indicated that children in both groups made similar improvements in articulation (both groups received articulation therapy); however, only the children who received myofunctional therapy demonstrated reduced tongue thrust.

More recently, Guisti Braislin and Cascella ${ }^{18}$ evaluated the impact of a marketed oral motor protocol ${ }^{12}$ on speech changes of four children with mild articulation disorders. Procedures followed the steps outlined in the therapy manual, so each treatment session began with gross body movements ("whole body wake-ups"), trunk and jaw stabilization followed by facial stimulation ("face wakeups"), and finally, practice on oral motor exercises. Each child received 7 weeks of treatment with an average of two sessions per week. Comparison of pretest and post-test Goldman-Fristoe Test of Articulation-2 ${ }^{19}$ (American Guidance Service, Circle Pines, $\mathrm{MN}$ ) raw scores indicated no change in children's speech as a result of the oral motor intervention.

It is surprising that so few empirical investigations of NSOMEs have been conducted, given the wealth of marketed products and the frequency with which these procedures are applied. ${ }^{20}$ Further, most of the studies just cited used myofunctional therapy and focused only on distorted sibilant production, yet NSOMEs typically target multiple speech errors across different places and manners of production. Finally, the existing literature does not provide any insight into the relative efficacy of oral motor therapy compared with speech-based interventions. For these reasons, the current investigation sought to evaluate the relative efficacy of NSOMEs versus PT on speech sound learning in a group of children with phonological/articulatory disorders (PADs).

\section{METHODS}

\section{Participants}

Ten subjects, ages $3 ; 3$ to $6 ; 3$ years with an average age of $4 ; 3$ years, were recruited through newspaper advertisements and flyers. Participants were required to use English as their primary language, have normal oral structures, and pass a pure tone hearing screening. The procedures used in this study were approved by the Indiana University Bloomington Campus Committee for the Protection of Human Subjects Institutional Review Board. Each child's parent or legal guardian signed a statement of informed consent prior to initiation of testing. Table 1 lists the participant characteristics.

All participants were assessed over the course of two 1-hour sessions. The evaluation included a pure tone hearing screening at $25 \mathrm{~dB}$ for octave frequencies between 250 and $8000 \mathrm{~Hz}$, an informal volitional oral motor (VOM) test, the Goldman-Fristoe Test of Articulation-2 ${ }^{19}$ (GFTA-2), an oral mechanism examination, the Peabody Picture Vocabulary Test-3 ${ }^{21}$ (PPVT-3; American Guidance Service, Circle Pines, MN), a nonword repetition task, ${ }^{22}$ and the collection of acoustic and language samples. Children who had standard scores below 85 on the GFTA-2 were considered to have PAD and, therefore, were eligible for further evaluation for potential entry into the study. All children with PAD completed the Clinical Evaluation of Language Fundamentals-Preschool (The Psychological Corporation, San Antonio, TX $)^{23}$ and a 
Table 1 Summary of Participant Characteristics

\begin{tabular}{|c|c|c|c|c|c|c|c|c|c|c|}
\hline $\begin{array}{l}\text { Subject } \\
\text { No. }\end{array}$ & $\begin{array}{l}\text { Subject } \\
\text { No. of } \\
\text { Match }\end{array}$ & $\begin{array}{l}\text { Age } \\
(\mathrm{mo})\end{array}$ & Sex & $\begin{array}{l}\text { GFTA-2 } \\
\text { Standard } \\
\text { Score }\end{array}$ & $\begin{array}{l}\text { PPVT-3 } \\
\text { Standard } \\
\text { Score }\end{array}$ & $\begin{array}{l}\text { VOM } \\
\text { Score } \\
\text { (max. 171) }\end{array}$ & $\begin{array}{l}\text { No. of Sounds } \\
\text { Missing from } \\
\text { Inventory }\end{array}$ & $\begin{array}{l}\text { NSOME } \\
\text { Target }\end{array}$ & $\begin{array}{l}\text { Production } \\
\text { Target }\end{array}$ & $\begin{array}{l}\text { Control } \\
\text { Sound }\end{array}$ \\
\hline 1 & 4 & 44 & $M$ & 83 & 120 & 150 & 10 & $\mathrm{t} \int$ & $\theta$ & $r$ \\
\hline 2 & 7 & 74 & $M$ & 60 & 119 & 109 & 3 & $\theta$ & $r$ & $\mathrm{t} \int$ \\
\hline 3 & 5 & 48 & $\mathrm{M}$ & 84 & 122 & 149 & 4 & k & $\theta$ & * \\
\hline 4 & 1 & 55 & $\mathrm{~F}$ & 67 & 92 & 102 & 6 & $\theta$ & $\mathrm{t} \int$ & $r$ \\
\hline $5^{\dagger}$ & 9 & 39 & $M$ & 69 & 112 & $\mathrm{~N} / \mathrm{A}$ & 10 & $\theta$ & k & $\mathrm{t} \int$ \\
\hline 6 & - & 75 & $\mathrm{M}$ & 80 & 96 & 170 & 4 & $\mathrm{~s}$ & $r$ & $\mathrm{t} \int$ \\
\hline 7 & 2 & 42 & $\mathrm{M}$ & 86 & 95 & 148 & 3 & $r$ & $\theta$ & z \\
\hline 8 & - & 48 & $\mathrm{M}$ & 67 & 97 & 112 & 9 & $\theta$ & $g$ & $\int$ \\
\hline 9 & 5 & 48 & $M$ & 69 & 85 & 138 & 7 & k & $\theta$ & $\int$ \\
\hline 10 & - & 65 & $\mathrm{~F}$ & 81 & 109 & 150 & 2 & $\ddagger$ & & \\
\hline
\end{tabular}

${ }^{*}$ No control sound was used for this subject because all other erred sounds either were emerging or were cognates of the treated sounds.

${ }^{\dagger}$ Subject 5 did not complete the VOM test so that score is not available (N/A).

${ }^{\ddagger}$ Subject 10 was removed from the experiment prior to participation in treatment phase because she obtained the targeted sound during baseline probing.

GFTA-2, Goldman-Fristoe Test of Articulation-2;19 PPVT-3, Peabody Picture Vocabulary Test-3;20 VOM, volitional oral movements; NSOME, nonspeech oral motor exercise.

200-word sound probe that provided opportunities for multiple productions of all English consonants in all word positions. Each participant's responses were recorded online by a graduate student in speech-language pathology, using broad phonetic transcription, and probe administration was videotaped for reliability purposes. A second graduate clinician independently transcribed the sound probe from the video recording. Any disagreement in transcription between the clinicians was resolved by consensus.

Children were enrolled in the treatment protocol if they had production errors on at least three unrelated sounds from their inventory as determined from the 200 -word probe. Sounds were considered linguistically unrelated if they differed from one another by at least one of the features of place and manner. This requirement was instituted to maximize the independence of treatment effects, as described next.

\section{Treatment Procedures}

An alternating treatment design was used to evaluate the effects of NSOMEs and PT on children's sound acquisition. NSOME treatment was provided on one sound, PT was administered for a second, linguistically unrelated sound, and a third sound was monitored as a control for nonexperiment effects. Treatment targets included at least one sound that was omitted from the child's inventory and a second sound that either was omitted or constrained such that it was only produced in a single word position or in a single lexical context. The control sound had to come from the inventory of omitted phonemes that were linguistically unrelated to either treatment target. For example, subject 1 received PT on $/ \theta /$, oral motor therapy on $/ \mathrm{t} \int /$, and $/ \mathrm{r} /$ served as the control sound (Table 1). When possible, children were paired such that one child received NSOMEs on sound A and PT on sound B, whereas a second child received PT on sound A and NSOMEs on sound B (e.g., S s 1\&4, 2\&7). This pairing was accomplished for seven of the children who participated in the treatment study. Additionally, targets were chosen to minimize interference of one sound on learning of the other sound.

Prior to treatment onset, baseline data were collected via sound-specific probes for each child's production of the treatment and control sounds. These probes also were used during treatment to test for generalization to nontreated words as described later. If there was any change in the child's production of a treatment or control sound during baseline 
testing, additional data were collected to determine if the change constituted random variation or learning. Only subject 10 demonstrated a change in production of the targeted oral motor sound during baseline testing and so she was eliminated from further participation. The remaining nine subjects maintained a stable baseline across three administrations of the probe words prior to treatment onset, and all completed the study.

Each of the nine subjects participated in both NSOME treatment and traditional PT during each therapy session. Sessions typically lasted 60 minutes each and occurred twice weekly with at least 1 day of no treatment separating the two sessions. Order of treatment was randomly determined such that either NSOME or traditional PT treatment occurred first for half the sessions, on average. Each treatment type lasted for 20 to 30 minutes per session with a 10 -minute play break in the middle. There were two main phases for both treatment types: imitation and spontaneous production. Feedback was provided on a continuum that ranged from continuous to variable. During the imitation phase, a model was provided to the participant and then feedback was provided. For PT, whole-word models were presented, and for NSOMEs, a model of the exercise was provided. Initially, during the continuous feedback phase, the child received feedback following each production. As the child increased the number of accurate productions to 15 accurate productions out of every 20 trials across three sets of trials, the feedback was decreased to variable reinforcement, which provided feedback following, on average, every three responses. Once the child reached criterion on 15 of 20 correct productions across three consecutive sets of 20 , the child moved to spontaneous production in response to the picture stimuli. Again, continuous feedback was provided, followed by the variable feedback as the child met the criterion for progression through the stages. The treatment words or NSOMEs were changed once the child produced accurate responses on 18 of 20 trials, across three sets, with variable reinforcement.

Progress in treatment was monitored with the use of sound-specific generalization probes that tested the child's production of the target sound and its cognate in all word positions on 30 untreated words. The criterion for termination of treatment was 20 correct productions out of 30 trials on the generalization probe, or completion of 20 treatment sessions, whichever was achieved first.

Sound-specific NSOMEs were selected from marketed sources. ${ }^{5,24}$ Although NSOMEs typically are used in conjunction with PT, such a design prevents evaluation of the independent benefit of each treatment type. For that reason, NSOMEs were trained independently of PT in this protocol.

NSOME treatment began with various resistance exercises and activities that required the child to move around within the treatment room (2 to 3 minutes) and facial stimulation that included having the child pat and stroke his or her face ( 2 to 3 minutes). Warm-up activities were followed by 100 trials of three different NSOMEs, such as stroking the center of the tongue with a tongue depressor, applying a resistance against the tongue, and lifting the tongue tip to the alveolar ridge. All exercises were modeled by the clinician until the child reached the criterion to progress to spontaneous production. Feedback and criteria for treatment changes were administered as described previously.

PT sessions included 100 trials of three consonant vowel consonant (CVC) stimuli in which $\mathrm{C}_{1}$ was the treatment target and $\mathrm{VC}_{2}$ varied. Targets were presented with picture stimuli, and in the initial imitation phase of treatment, participants were provided with a model for every trial. Again, feedback and criteria for stimulus presentation were consistent with methods described earlier.

\section{Data Analysis}

The difference in percentage correct production of target sounds from pretreatment to posttreatment was calculated from the soundspecific generalization probes (i.e., NSOMEtreated sound and PT sound) for all subjects except subject 2. Due to teasing from some children, subject 2 practiced production of the NSOME sound target for six consecutive evenings (as reported by his parents) before the final treatment session. For that reason, the 


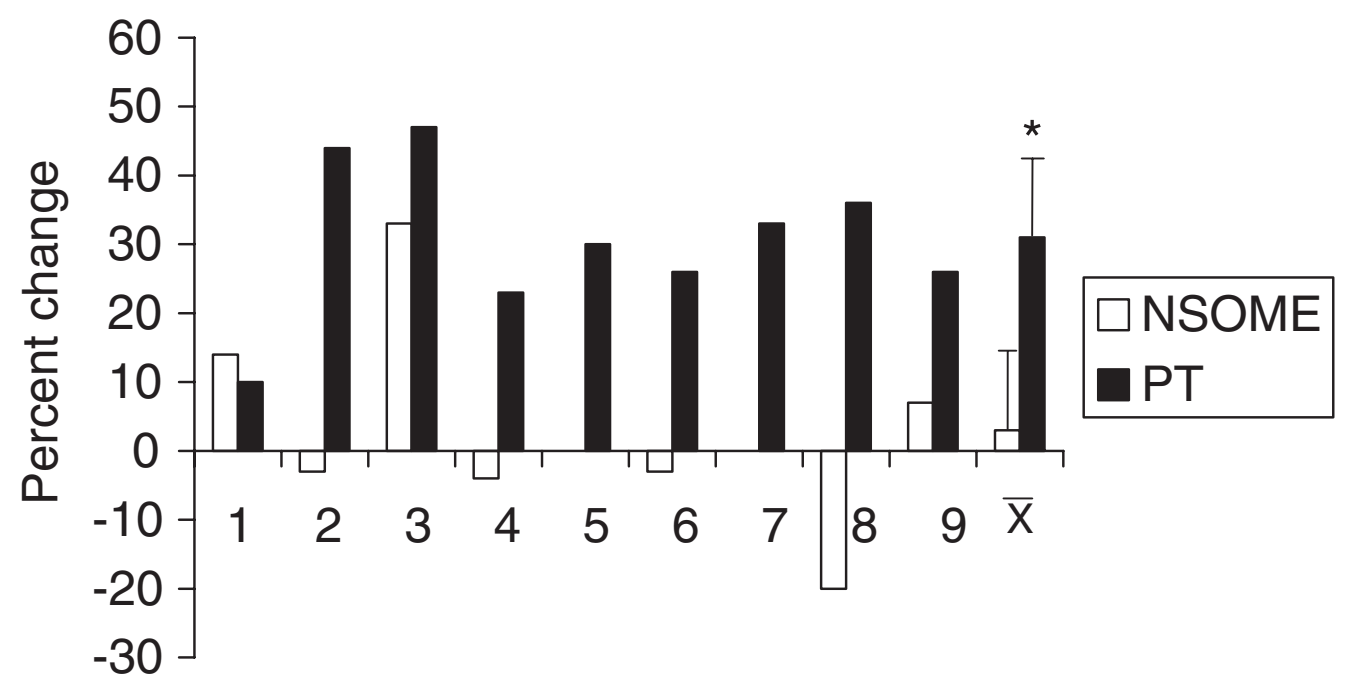

\section{Subject number}

Figure 1 Percentage change in target sound production for each subject as measured by the difference between post-treatment and pretreatment generalization probes. White bars represent the nonspeech oral motor exercise (NSOME) change, and dark bars denote changes with production treatment (PT). Bars below the zero line indicate fewer correct productions of the target sound after treatment compared with the pretreatment evaluation. Bars that extend above the zero line represent improved performance on target sound production, as indexed by the generalization probe, after treatment compared with pretreatment accuracy. The final bars represent the mean percentage change and standard deviations for each treatment type. As noted by the asterisk, there was a significant difference in the effect of the two treatments.

NSOME and PT generalization probes from the second to last session were used instead of the final probe data; however, results from both sessions are presented. A paired $t$ test was used to compare the percentage change (i.e., posttreatment minus pretreatment) in correct production for the two treatment types. In addition, qualitative evaluation of the impact of NSOMEs on PT was undertaken. This analysis compared the percentage correct of treatment word production when NSOMEs preceded PT to this metric obtained when PT was administered before NSOMEs. If oral motor exercises facilitate PT, one would expect that PT would improve when NSOMEs were presented first. Finally, the relation between pretreatment oral skills, as measured by the VOM scores, to changes in sound production was described with the expectation that if NSOMEs improve oral motor skill, then children with low ability should benefit from NSOMEs as indexed by increased articulatory accuracy.

\section{RESULTS}

The PT yielded a 30\% increase, on average, in sound accuracy relative to pretreatment production compared with a $3 \%$ change that resulted from NSOMEs. This difference was statistically significant $\left(t_{8}=4.7549\right.$; $p=0.0014)$ when subject 2's second to last generalization probe was used to evaluate treatment efficacy but lacked statistical significance if his final probes were used $\left(\mathrm{t}_{8}=-2.23\right.$; $p=0.056)$. No changes in control sound production were noted for any subjects. As seen in Fig. 1, eight of nine subjects (subjects 2 to 9) made greater changes in the sound targeted by PT compared with the sound that was treated with NSOMEs. Again, this result is valid if subject 2's last probe is not used for comparison. Inclusion of his final probe would suggest that NSOMEs provided him with greater benefit than PT (73\% for final NSOME versus $33 \%$ for final PT).

No facilitative effect of NSOMEs was observed on production targets. Analysis of 
treatment data revealed that children were as likely to show improvements in production targets when NSOME therapy came before PT (51\% of sessions with this order) as when treatment order was reversed $(54 \%$ of production-first sessions). Four participants, $(2,4,6$, and 8$)$, exhibited negative change in accuracy following NSOME therapy, indicating that their performance on the sound generalization probe actually declined after participating in NSOME treatment.

Finally, NSOMEs did not appear to provide children with increased oral motor skill. The children with the lowest VOM scores (subjects 2, 4, and 8) did not show any differential gains in speech production compared with children with higher pretreatment VOM scores.

\section{DISCUSSION}

Results of this investigation do not support the use of NSOMEs as an effective procedure for improving speech sound production. Only subject 1 showed a modest advantage of NSOMEs over PT (14\% increase in production accuracy for NSOMEs compared with $10 \%$ change from PT); however, six of the other subjects showed no change or reductions in sound accuracy after 20 NSOME treatment sessions. By comparison, all subjects made some gains when traditional articulation training was administered.

These results for the NSOME treatment are consistent with findings from other motor systems (e.g., limbs) in which training of a part of a movement sequence does not facilitate learning of the integrated behavior. ${ }^{12-14}$ As with those studies, the current investigation continues to show that acquisition of highly complex organized behaviors such as speech cannot be mastered by treating subcomponents of the movement. Other rationales for using NSOMEs also were not supported by the current study. That is, there was no evidence of increased oral orientation or attention that resulted from NSOMEs, although it is not clear how this issue can be assessed directly in pediatric populations. Evaluation of the influence of NSOMEs on PT provides no indication of a facilitative effect of these exercises on speech sound production; however, this result needs further investigation because the NSOMEs targeted a sound that was linguistically distinct from the PT target. As such, a facilitative effect may not be expected. Despite this attempted control, there were some NSOMEs that were prescribed for both target sounds (e.g., resistance against a tongue depressor in superior or anterior movement), and no facilitative effect on PT occurred under these conditions. Finally, if NSOMEs increase articulator strength or coordination, one would predict that the subjects with the lowest pretreatment VOMs would show gains from NSOMEs, but the opposite pattern emerged. Subjects with the lowest VOM scores had negative gains in production of the NSOME-treated sound, thus suggesting that these procedures did not improve movement control.

There are many reasons to question the use of NSOMEs as a means of remediating speech disorders, including theoretical (i.e., transfer of training), ${ }^{7,12-14}$ anatomical (i.e., histology of speech articulators), ${ }^{3,8}$ and empirical concerns (i.e., data from the current study and others). Because there appears to be a critical period for speech sound acquisition, ${ }^{24}$ and because many other treatments have been proven to improve phonetic inventory development, ${ }^{25-27}$ strong evidence for the efficacy of NSOMEs should be demonstrated before the inclusion of such exercises in PAD therapy regimens.

\section{ACKNOWLEDGMENT}

This research was supported by NIH grant DC-R01-04575.

\section{REFERENCES}

1. Lof GL. Logic, theory and evidence against the use of non-speech oral motor exercises to change speech sound productions. Paper presented at: annual convention of the American Speech Language Hearing Association; November 2006; Miami Beach, FL

2. Marshalla PR. Oral motor treatment vs non-speech oral motor exercises. Oral Motor Inst; 2008. Available at: http://www.oralmotorinstitute.org/ mons/v2n2_marshalla.html. Accessed July 8, 2008 
3. Weismer G. Philosophy of research in motor speech disorders. Clin Linguist Phon 2006;20(5): 315-349

4. Rosenfeld-Johnson S. Oral-Motor Exercises for Speech Clarity. Tucson, AZ: Talk Tools; 2001

5. Chapman Bahr D, Hillis AE. Oral Motor Assessment and Treatment. Boston, MA: Allyn \& Bacon; 2001

6. Forrest K. Are oral-motor exercises useful in the treatment of phonological/articulatory disorders? Semin Speech Lang 2002;23:15-25

7. Clark H. Neuromuscular treatments for speech and swallowing: a tutorial. Am J Speech Lang Pathol 2003;12:400-415

8. Bahr D. A topical bibliography on oral motor assessment and treatment. Oral Motor Inst; 2008. Available at: www.oralmotorinstitute.org/mons/ v2n1_bahr.html. Accessed July 11, 2008

9. Strode R, Chamberlain C. Easy Does It for Articulation: An Oral Motor Approach. East Moline, IL: LinguiSystems; 1997

10. Bunton K. Speech versus nonspeech: Different tasks, different neural organization. Semin Speech Lang 2008;29:267-275

11. Clark H. The role of strength training in speech sound disorders. Semin Speech Lang 2008;29: 276-283

12. Adams JA. Historical review and appraisal of research on the learning, retention, and transfer of human motor skills. Psychol Bull 1987;10:41-74

13. Naylor JC, Briggs GE. Effects of task complexity and task organization on the relative efficiency of part and whole training methods. J Exp Psychol 1963;65:217-224

14. Wightman D, Lintern G. Part-task training for tracking and manual control. Hum Fac 1985;27: 267-283

15. Ruscello DM. Nonspeech oral motor treatment issues related to children with developmental speech sound disorders. Lang Speech Hear in Schools 2008;39:380-391
16. Overstake C. Investigation of the efficacy of a treatment program for deviant swallowing and allied problems, part II. Int J Myology 1976;2: 1-6

17. Christensen M, Hanson M. An investigation of the efficacy of oral myofunctional therapy as a precursor to articulation therapy. J Speech Hear Disord 1981;46:160-167

18. Guisti Braislin MA, Cascella PW. A preliminary investigation of the efficacy of oral motor exercises for children with mild articulation disorders. Int J Rehabil Res 2005;28:263-266

19. Goldman R, Fristoe M. The Goldman-Fristoe Test of Articulation-2. Circle Pines, MN: American Guidance Service; 2000

20. Lof GL, Watson MM. A nationwide survey of nonspeech oral motor exercise use: Implications for evidence-based practice. Lang Speech Hear in Schools 2008;39:392-407

21. Dunn LM, Dunn LM. Peabody Picture Vocabulary Test. 3rd ed. Circle Pines, MN: American Guidance Service; 1997

22. Dollaghan C, Campbell TF. Nonword repetition and child language impairment. J Speech Lang Hear Res 1998;41:1136-1146

23. Wiig E, Secord W, Semel E. Clinical Evaluation of Language Fundamentals-Preschool. San Antonio, TX: The Psychological Corp; 1992

24. Pehde H, Geller A, Lechner B. The Complete Oral-Motor Program for Articulation. East Moline, IL: LinguiSystems; 1996

25. Shriberg LD, Gruber FA, Kwiatkowski J. Developmental phonological disorders. III: Long-term speech-sound normalization. J Speech Hear Res 1994;37:1151-1177

26. Gierut JA. Treatment efficacy: functional phonological disorders in children. J Speech Lang Hear Res 1998;41:S85-S100

27. Rvachew S, Nowak M. The effect of targetselection strategy on phonological learning. J Speech Lang Hear Res 2001;44:610-623 